\title{
Multimodal Interferometer Based on a Suspended Core Fiber for Simultaneous Measurement of Physical Parameters
}

\author{
Sergio Rota-Rodrigo, Manuel López-Amo, Senior Member, IEEE, Member, OSA, Jens Kobelke, Kay Schuster, \\ José Luis Santos, and Orlando Frazão, Senior Member, IEEE, Member, OSA
}

\begin{abstract}
In this study, a multimodal interferometer based on a suspended core photonic crystal fiber (PCF) for simultaneous strain and temperature measurements is proposed. The structure is also employed for angle measurements. The sensor comprises a 3-mm-suspended core PCF between SMFs and is based on the combination of two multimodal interferences with different frequency fringe patterns. The interferometric patterns show different sensitivity responses to strain and temperature. Through a low-pass frequency filtering of the detected spectrum, the wavelength shift of the two patterns can be measured allowing the discrimination of strain and temperature effects with resolutions of $0.45{ }^{\circ} \mathrm{C}$ and 4.02 $\mu \varepsilon$, respectively. The sensor is also characterized for angle measurements showing a maximum sensitivity of $9.17 \mathrm{pm} /{ }^{\circ}$ in the range from $0^{\circ}$ to $90^{\circ}$. It is demonstrated that with this sensing structure is possible to obtain simultaneous measurement of bend angle and temperature with resolutions of $1.69^{\circ}$ and $0.92{ }^{\circ} \mathrm{C}$, respectively.
\end{abstract}

Index Terms-Curvature sensor, fiber optic sensor, photonic crystal fiber, strain sensor, temperature sensor.

\section{INTRODUCTION}

$\mathbf{O}$ PTICAL fiber sensors have been thoroughly investigated due to their competitive application in the monitoring and control of some physical parameters. In particular, temperature, strain and curvature sensors have grown significantly in importance for health monitoring of aerospace, marine or civil structures, and in fabrication processes, among others [1], [2]. A fiber optic sensor presents relevant advantages such as its

Manuscript received August 4, 2014; revised October 6, 2014 and October 31, 2014; accepted November 24, 2014. Date of publication December 1, 2014; date of current version April 29, 2015. This work was supported by the Spanish Government Projects TEC2010-20224-C02-01 and TEC2013-47264 C2-2-R, the European Cooperation in Science and Technology Action TD1001, the European FEDER funding, ECOAL-MGT SUDOE project, and Project "NORTE-07-0124-FEDER-000058" that was financed by the North Portugal Regional Operational Programme (ON.2-O Novo Norte), under the National Strategic Reference Framework, the European Regional Development Fund, and by national funds, through the Portuguese Funding Agency, Fundação para a Ciência e a Tecnologia.

S. Rota-Rodrigo and M. López-Amo are with the Department of Electronic and Electronic Engineering, Universidad Pública de Navarra, E-31006 Pamplona, Spain (e-mail: Sergio.rota@unavarra.es; mla@unavarra.es).

J. Kobelke and K. Schuster are with the Institute of Photonic Technology, D-07745 Jena, Germany (e-mail: jens.kobelke@ipht-jena.de; kay.schuster@ ipht-jena.de).

J. L. Santos and O. Frazão are with the Institute for Systems and Computer Engineering of Porto, 4169-007 Porto, Portugal, and the Faculdade de Ciências da Universidade do Porto, 4169-007 Porto, Portugal (e-mail: josantos@fc.up.pt; ofrazao@inescporto.pt).

Color versions of one or more of the figures in this paper are available online at http://ieeexplore.ieee.org.

Digital Object Identifier 10.1109/JLT.2014.2375896 immunity to electromagnetic interference, small size, reliability, remote sensing ability and multiplexing capability.

Multimode interferometers (MMI) have been extensively considered with standard multimode fibers for sensing of curvature [3], strain, temperature [4] as well as for simultaneous parameter measurements [5]. The appearance of photonic crystal fibers (PCFs) has allowed continuing the investigation of MMI associated now to novel fiber structures with distinct characteristics.

PCFs consist of a regular array of air-holes along the entire length of a thread of pure silica glass. These optical fibers present diverse improved features compared with common single-mode fibers (SMFs) [6].

There are different ways to achieve strain sensors based on PCFs. There are strain sensors based on multimode interference by using multimode PCF or by tapering sections of PCF [7], [8]. Other reported strain sensors rely on birefringent PCFs by detecting wavelength changes in a Hi-Bi PCF Sagnac interferometric configuration [9], or by detecting amplitude variations in an in-reflection Hi-Bi PCF polarimetric interferometric sensor [10]. Also, it has been proposed strain sensor layouts based on considering twin core PCFs operating as Mach Zehnder interferometers [11].

Furthermore, there are sensing structures for simultaneous strain and temperature measurement based on the combination of PCF interferometers with fiber Bragg gratings (FBGs) or long period gratings (LPG), in order to discriminate both physical parameters. In [12] a PCF modal interferometer in combination with a FBG was presented, with report of resolutions of $\pm 0.27^{\circ} \mathrm{C}$ and $\pm 9.1 \mu \varepsilon$. Other authors proposed a PCF modal interferometer combined with a LPG in a layout that permitted resolutions of $\pm 1.5^{\circ} \mathrm{C}$ and $\pm 5.2 \mu \varepsilon$ for temperature and strain, respectively [13].

Other dual parameter sensor configurations were based on the detection of different interference components showing different sensitivities to strain and temperature. In [14] a new design based on a clover geometry PFC in a fiber loop mirror (FLM) topology showed resolutions of $\pm 2{ }^{\circ} \mathrm{C}$ and $\pm 11 \mu \varepsilon$ for these two measurands. Also in [15] a Hi-Bi PCF was incorporated into a FLM layout resulting in of $\pm 1.5^{\circ} \mathrm{C}$ and $\pm 4.7 \mu \varepsilon$.

The measurement of bend angles is highly interesting for structural health monitoring and mechanical engineering. In [16] an inclinometer based on a modal interferometer by using a hollow core PCF was presented, with an operation range of \pm 45 deg. Furthermore, in [17] a Mach-Zehnder interferometer 


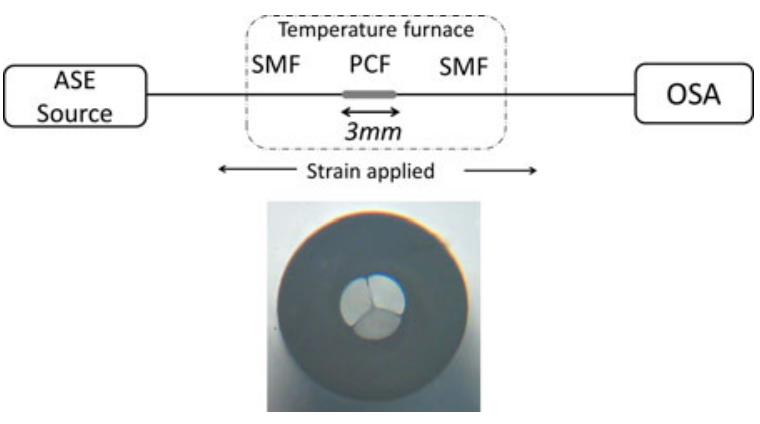

Fig. 1. Schematic of the strain sensor system (top) and photo of the suspendedcore PCF cross-section (bottom).

based on a twin core PCF was demonstrated for angle measurements in order to isolate the bend direction.

In this work are proposed and investigated two sensing structures for strain-temperature and angle-temperature measurements, both based on the same suspended core PCF. The structures comprises a length of suspended core PCF between SMF's and is based on the combination of two multimodal interferograms with different fringe patterns [18]. We report resolutions of $\pm 4.02 \mu \varepsilon$ and $\pm 0.45^{\circ} \mathrm{C}$ for the simultaneous strain-temperature sensor, and of $\pm 1.69 \mathrm{deg}$ and $\pm 0.92{ }^{\circ} \mathrm{C}$ for the simultaneous angle-temperature sensor [19].

\section{EXPERIMENTAL SETUP}

The sensing principle derives from the analysis of the interferogram that results from the combination of two different multimodal interferometric patterns originated by introducing a $3 \mathrm{~mm}$ length suspended-core PCF between two SMFs (see Fig. 1). This PCF presents an asymmetric core with $\sim 2.4 \mu \mathrm{m}$ diameter enclosed by three large holes, showing a multimode behavior. The shorter length of the sensor and its configuration in transmission, makes it more versatile than sensors based on a fiber loop mirror in which the length usually is in the order of centimeters [14], [15].

The SMF-PCF splices were done using a splice machine (Fitel S182PM) in manual mode, with an arc-power of 60 (splicer parameter) and an arc-duration of $650 \mathrm{~ms}$. These splice parameters allows the repeatability of the sensor.

The experimental configuration included an erbium-doped fiber amplifier (EDFA, Fiberamp-BT 1400) operating as a broadband optical source and an optical spectrum analyzer (OSA, Advantest Q8384) with a maximum resolution of $10 \mathrm{pm}$. The fiber was fixed on two translation stages, separated $50 \mathrm{~cm}$, in order to apply strain by moving one of the stages while the other remains fixed.

Fig. 2 shows the transmission spectrum of the sensing head (dark line). Two different frequency components can be appreciated, associated to high and low frequency fringe patterns. The low frequency one comes from the birefringence generated by the asymmetric core of the PCF, while the high frequency pattern results from the interference of the modes that propagates in the sensing head fiber which shows a multimode behavior.

Fig. 3 gives the fast Fourier transform of the transmission spectrum shown in Fig. 2, evidencing the two frequency com-

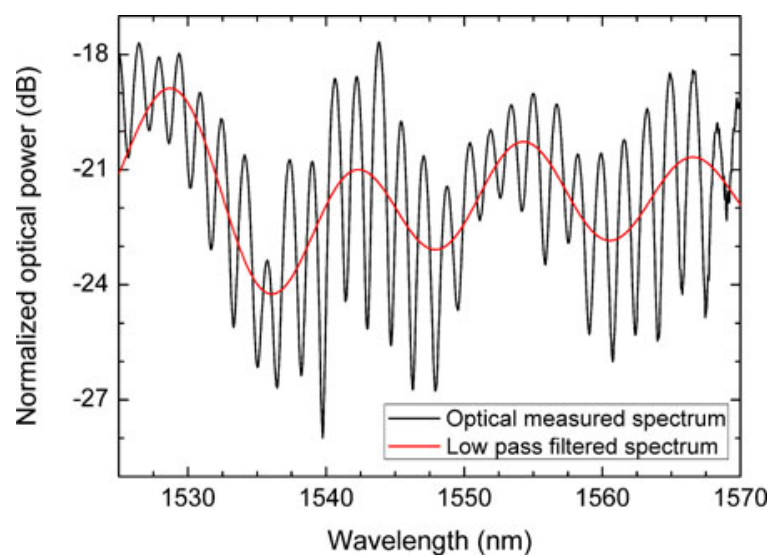

Fig. 2. Optical transmission spectra of the sensor at room temperature when no external force is applied (dark line), and its low frequency component (red line).

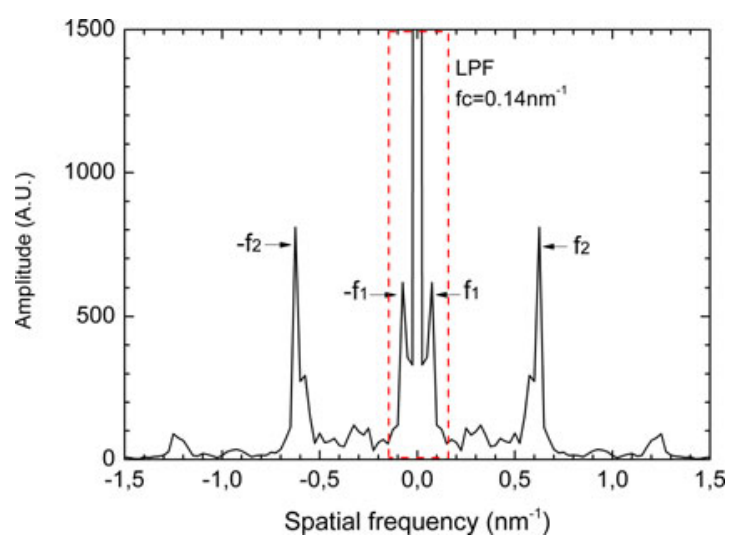

Fig. 3. Fast Fourier transform of the optical transmission spectra given in Fig. 2 (dark line).

ponents, $\mathrm{f}_{1}$ and $\mathrm{f}_{2}$, which correspond to the low and high frequency fringe patterns, respectively. The low frequency component present in Fig. 2 was obtained using a digital low-pass filter with a cut-off frequency of $0.14 \mathrm{~nm}^{-1}$. The presence of two clearly periodic interference, allows the repeatability of the sensor in comparison with those based on non-periodic interference [12], [14].

\section{SimultanEOUS STRAIN AND TEMPERATURE MEASUREMENTS}

For the strain characterization of the sensing head a measurement from 0 to $2000 \mu \varepsilon$ with steps of $200 \mu \varepsilon$ was carried out. Fig. 4(a) shows the wavelength shift of each interference pattern. This characterization was carried out by tracking a fringe maximum of the spectrum of each interference pattern. The low-frequency interferogram had a considerable major sensitivity, $-3.16 \mathrm{pm} / \mu \varepsilon$, in comparison with the $+0.34 \mathrm{pm} / \mu \varepsilon$ of the high-frequency one. Fig. 4(b) shows the evolution with strain of the low-frequency spectrum.

Once the strain response was characterized, a temperature study was carried out. For this purpose, a measurement from $40^{\circ} \mathrm{C}$ to $70^{\circ} \mathrm{C}$ with steps of $5^{\circ} \mathrm{C}$ was conducted. Fig. 5(a) shows 


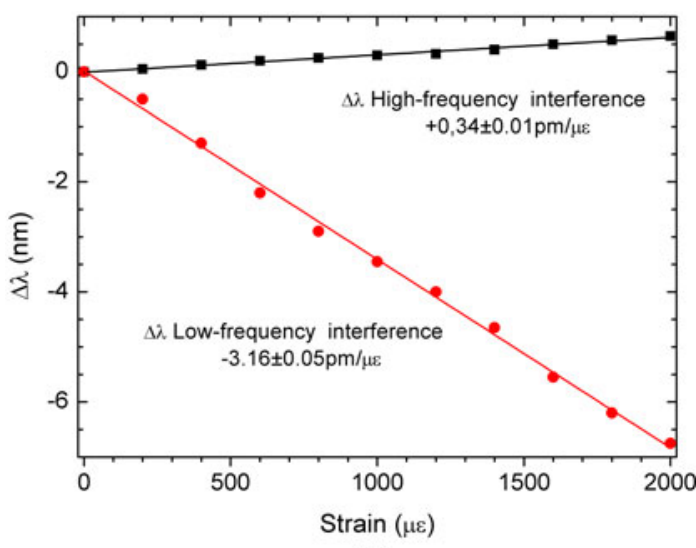

(a)

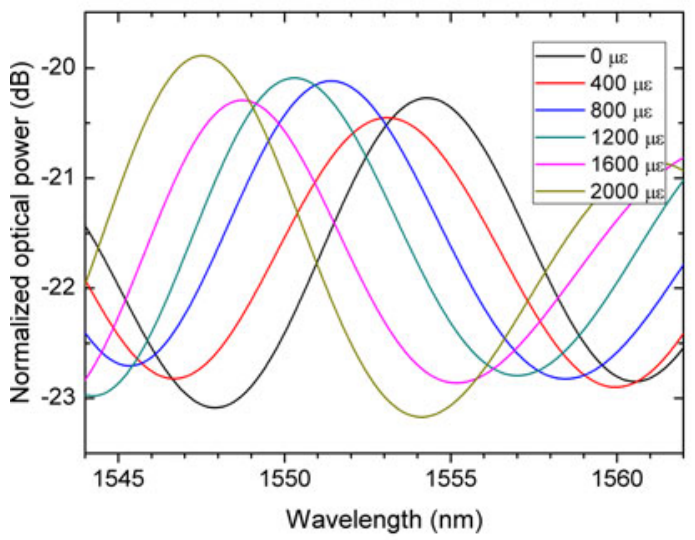

(b)

Fig. 4. Characterization of the wavelength shift with strain (a), and spectral evolution of the low-frequency interferometric pattern (b).

that in this case the high-frequency interferogram had a higher sensitivity $\left(25.30 \mathrm{pm} /{ }^{\circ} \mathrm{C}\right)$ compared with the low-frequency one $\left(-6.01 \mathrm{pm} /{ }^{\circ} \mathrm{C}\right)$. The unfiltered spectral evolution with temperature variations is shown in Fig. 5(b).

With the results obtained from the strain and temperature dependence of the two interferometric patterns it can be written:

$$
\begin{aligned}
\Delta \lambda_{\text {Low }- \text { freq }} & =-6.01 \cdot \Delta T-3.16 \cdot \Delta \varepsilon \\
\Delta \lambda_{\text {High-freq }} & =25.30 \cdot \Delta T+0.34 \cdot \Delta \varepsilon
\end{aligned}
$$

where $\Delta \lambda_{\text {low }- \text { freq }}$ and $\Delta \lambda_{\text {High }- \text { freq }}$ are expressed in pm. These equations reveal it is possible to discriminate the strain and temperature effects by rearranging them in the following way:

$$
\left[\begin{array}{c}
\Delta T \\
\Delta \varepsilon
\end{array}\right]=\frac{1}{77.908} \cdot\left[\begin{array}{cc}
0.34 & 3.16 \\
-25.30 & -6.01
\end{array}\right]\left[\begin{array}{l}
\Delta \lambda_{\text {Low }- \text { freq }} \\
\Delta \lambda_{\text {High }- \text { freq }}
\end{array}\right]
$$

The temperature and strain measurement based on this approach show resolutions, $\delta T$ and $\delta \varepsilon$, which were calculated using the method presented in [20] considering that the OSA has a spectral resolution of $\delta_{\mathrm{OSA}}=10 \mathrm{pm}$. It turned out values of $\delta T=0.45^{\circ} \mathrm{C}$ and $\delta \varepsilon=4.02 \mu \varepsilon$, which compares favorably with values reported by other authors, as outlined in Table I.

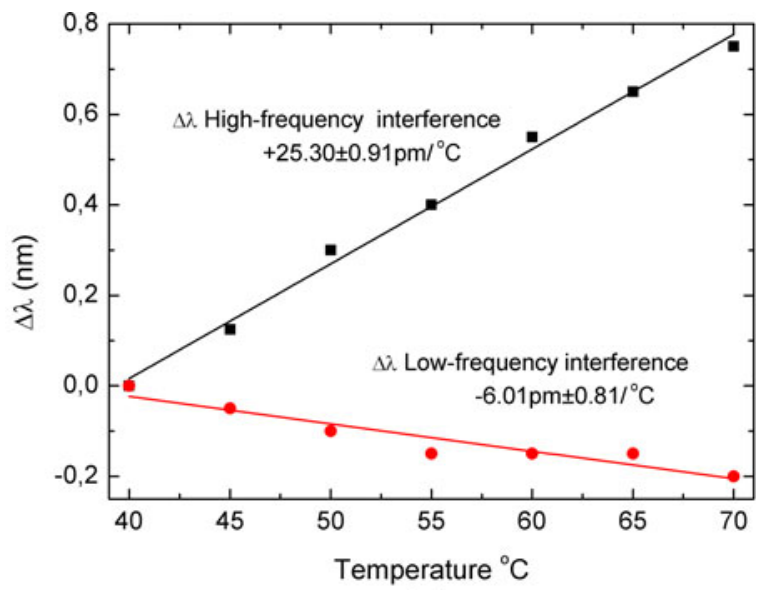

(a)

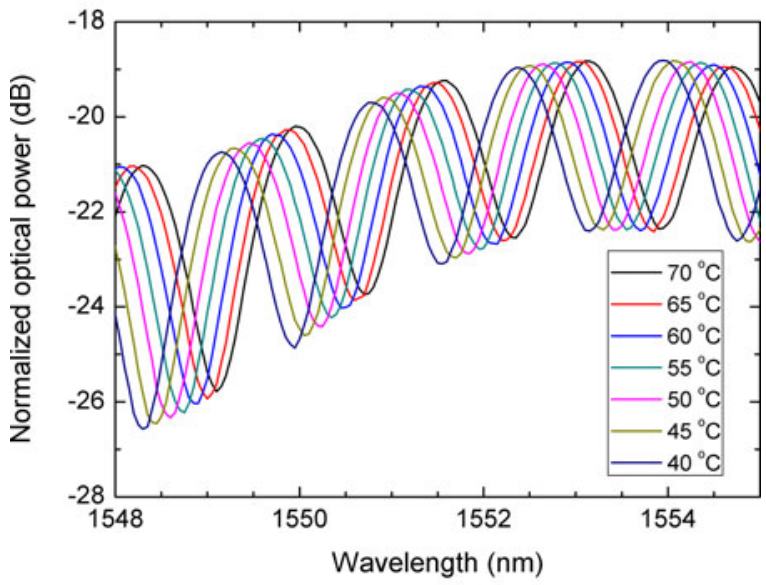

(b)

Fig. 5. Characterization of the wavelength shift with temperature of the two interferometric patterns (a), and evolution of the spectra with temperature (b).

TABLE I

VALUES FOR STRAIN AND TEMPERATURE RESOLUTIONS UNDER Simultaneous MEASUREMENT OBTAINED CONSIDERING OTHER Fiber OPTIC SENSING LAYOUTS

\begin{tabular}{lcccc}
\hline \hline Technology & $\delta \varepsilon$ & $\delta \mathrm{T}$ & Year & Ref. \\
\hline Modal interferometer + FBG & $9.1 \mu \varepsilon$ & $0.27^{\circ} \mathrm{C}$ & 2010 & {$[12]$} \\
Modal interferometer + LPG & $5.2 \mu \varepsilon$ & $1.5^{\circ} \mathrm{C}$ & 2012 & {$[13]$} \\
Clover PCF FLM & $11 \mu \varepsilon$ & $2.0^{\circ} \mathrm{C}$ & 2012 & {$[14]$} \\
Hi-Bi PCF FLM & $4.7 \mu \varepsilon$ & $1.5^{\circ} \mathrm{C}$ & 2010 & {$[15]$} \\
\hline \hline
\end{tabular}

\section{ANGLE MEASUREMENTS}

The sensor was also characterized for bend angle $(\alpha)$ measurements. An angle stepper was used in the range of -90 deg to +90 deg with steps of $30 \mathrm{deg}$ (Fig. 6). The angle was measured in relation to the two fixed points of the fiber (Fig. 6 in blue), with a separation of $7.5 \mathrm{~cm}$.

Fig. 7 shows the evolution of the low-frequency spectral interferogram with the angle applied, indicating an appreciable change in wavelength and amplitude. In order to overcome in the measurement attenuation effects it was decided to track the wavelength evolution, which is shown in Fig. 7(b). It can be 


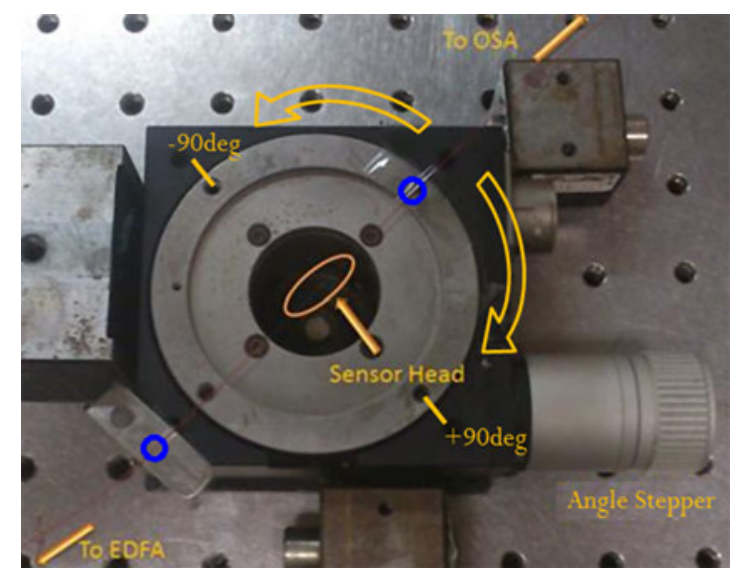

Fig. 6. Setup of the bend sensor with the angle stepper.

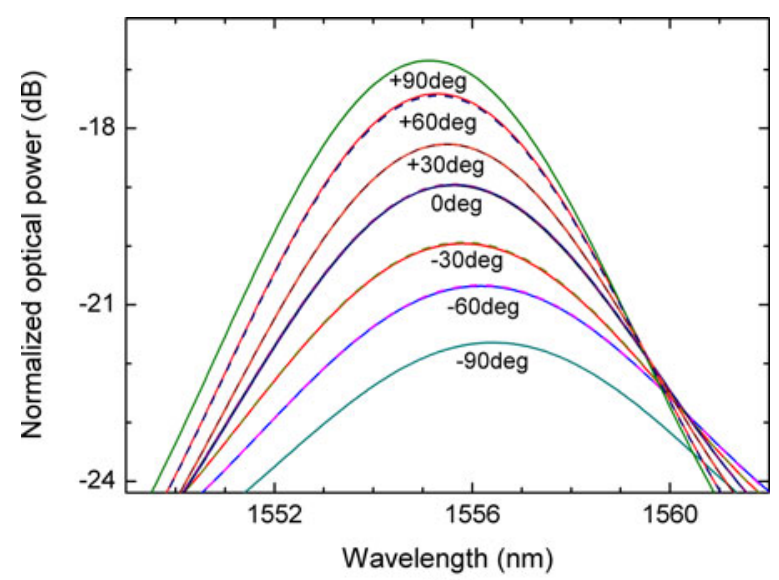

(a)

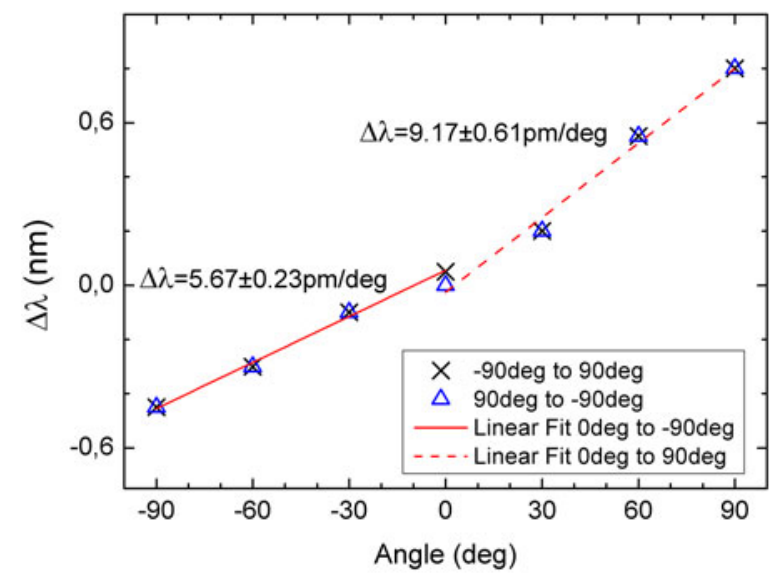

(b)

Fig. 7. Evolution of the low-frequency interferogram with angle (a) and characterization of the wavelength shift with curvature (b).

observed the sensor presents two regions with different sensitivities. This is attributed to the PCF asymmetric core and its birefringence. However, both regions evidenciate a linear behavior. The angular sensitivity of the sensor is dependent on the position of the fiber core with respect to the angle direction. From the date shown in Fig 7(b), a sensitivity value of

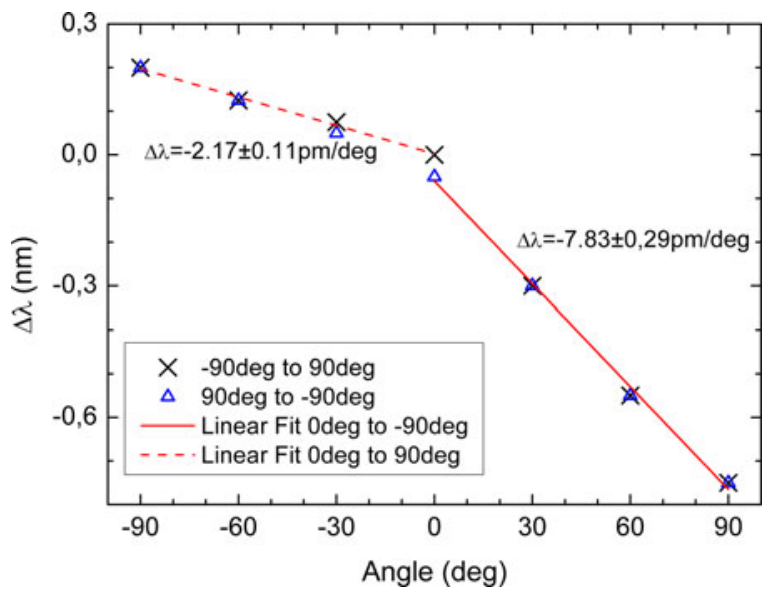

Fig. 8. Characterization of the wavelength shift of the high-frequency interferogram with curvature (b).

$5.67 \mathrm{pm} / \mathrm{deg}$ is derived for the range 0 to $-90 \mathrm{deg}$, while for the range 0 to $90 \mathrm{deg}$ it is $9.17 \mathrm{pm} / \mathrm{deg}$.

Likewise, the high-frequency spectral interferogram presents the same behavior (see Fig. 8), in this case with sensitivities of $-2.17 \mathrm{pm} / \mathrm{deg}$ and $-7.83 \mathrm{pm} / \mathrm{deg}$ in the ranges of 0 to $-90 \mathrm{deg}$ and 0 to $90 \mathrm{deg}$, respectively.

Due to the linear behavior of the sensor response in each angular zone, it is possible to configure the sensing head for simultaneous measurement of bend angle and temperature. For this purpose we used the characterization in the range from 0 to 90 deg which presents a higher sensitivity to angle variations. The wavelength variation (in pm) of the high and low frequency interferograms can be expressed as:

$$
\begin{aligned}
& \Delta \lambda_{\text {Low }- \text { freq }}=9.17 \cdot \Delta \alpha-6.01 \cdot \Delta T \\
& \Delta \lambda_{\text {High-freq }}=-7.83 \cdot \Delta \alpha+25.3 \cdot \Delta T
\end{aligned}
$$

This way the angle and temperature can be calculated by:

$$
\left[\begin{array}{c}
\Delta \alpha \\
\Delta \mathrm{T}
\end{array}\right]=\frac{1}{184.95} \cdot\left[\begin{array}{ll}
25.3 & 6.01 \\
7.83 & 9.17
\end{array}\right]\left[\begin{array}{l}
\Delta \lambda_{\text {Low }- \text { freq }} \\
\Delta \lambda_{\text {High }- \text { freq }}
\end{array}\right]
$$

Applying the same approach considered for the simultaneous measurement of strain and temperature, the resolution was evaluated resulting in values of $\delta \alpha=1.69 \mathrm{deg}$ and $\delta T=0.92{ }^{\circ} \mathrm{C}$, for curvature and strain, respectively

\section{CONCLUSIONS}

In conclusion, a simultaneous temperature-strain fiber optic sensor based on the combination of the characteristics of two interferograms with disctinct frequency fringe patterns was proposed and demonstrated. The sensing head was accomplished by using a three hole suspended core PCF operated in transmission. Each interferometric pattern presents different response to strain and temperature, allowing the simultaneous measurement of both parameters. Through a low-pass frequency filtering of the detected spectrum, the wavelength displacement of each interferogram can be measured allowing the discrimination of temperature and strain with resolutions of $0.45^{\circ} \mathrm{C}$ and $4.02 \mu \varepsilon$, respectively. Furthermore, the sensor was tested for angle 
measurements, with demonstration that a sensitivity of $9.17 \mathrm{pm} / \mathrm{deg}$ in the 0 to $90 \mathrm{deg}$ range is possible. On this line, results were presented indicating the feasibility of simultaneous measurement of bend angles and temperature with resolutions of $\pm 1.69 \mathrm{deg}$ and $\pm 0.92{ }^{\circ} \mathrm{C}$, respectively.

\section{REFERENCES}

[1] J. M. Lopez-Higuera, L. Rodriguez Cobo, A. Quintela Incera, and A. Cobo, "Fiber optic sensors in structural health monitoring," J. Lightw. Technol., vol. 29, no. 4, pp. 587-608, Feb. 2011.

[2] H. N. Li, D. S. Li, and G. B. Song, "Recent applications of fiber optic sensors to health monitoring in civil engineering," Eng. Struct., vol. 26, pp. 1647-1657, 2004.

[3] Y. Gong, T. Zhao, Y. Rao, and Y. Wu, "All-fiber curvature sensor based on multimode interference," IEEE Photon. Technol. Lett., vol. 23, no. 11, pp. 679-681, Jun. 2011.

[4] S. M. Tripathi, A. Kumar, R. K. Varshney, Y. B. P. Jumar, E. Marin, and J. Meunier, "Strain and temperature sensing characteristics of singlemode-multimode-single-mode structures," J. Lightw. Technol., vol. 27, no. 13, pp. 2348-2356, Jul. 2009.

[5] Q. Wu, A. M. Hatta, P. Wang, Y. Semenova, and G. Farrell, "Use of a bent single SMS fiber structure for simultaneous measurement of displacement and temperature sensing," IEEE Photon. Technol. Lett., vol. 23, no. 2, pp. 130-132, Jan. 2011.

[6] A. M. R. Pinto and M. Lopez-Amo, "Photonic crystal fibers for sensing applications," J. Sens., vol. 2012, 21 pages, 2012.

[7] J. Villatoro, V. P. Minkovich, and D. Monzon-Hernandez, "Temperatureindependent strain sensor made from tapered holey optical fiber," Opt. Lett., vol. 31, pp. 305-307, 2006.

[8] R. M. André, S. O. Silva, M. Becker, K. Schuster, M. Rothardt, H. Bartelt, M. B. Marques, and O. Frazão, "Strain sensitivity enhancement in suspended core fiber tapers," Photon. Sens., vol. 3, pp. 118-123, 2013.

[9] O. Frazao, J. M. Baptista, and J. L. Santos, "Temperature-independent strain sensor based on a Hi-Bi photonic crystal fiber loop mirror," IEEE Sens. J., vol. 7, no. 10, pp. 1453-1455, Oct. 2007.

[10] S. Rota-Rodrigo, M. Bravo, A. M. R. Pinto, and M. López-Amo, "An in-reflection strain sensing head based on a Hi-Bi photonic crystal fiber," Sensors, vol. 13, pp. 8095-8102, 2013.

[11] K. K. Qureshi, Z. Liu, H. Y. Tam, and M. F. Zia, "A strain sensor based on in-line fiber Mach-Zehnder interferometer in twin-core photonic crystal fiber," Opt. Commun., vol. 309, pp. 68-70, 2013.

[12] B. Dong, J. Hao, C. Y. Liaw, B. Lin, and S. C. Tjin, "Simultaneous strain and temperature measurement using a compact photonic crystal fiber inter-modal interferometer and a fiber Bragg grating," Appl. Opt., vol. 49, pp. 6232-6235, 2010.

[13] T. Li, X. Dong, C. C. Chan, L. Hu, and W. Qian, "Simultaneous strain and temperature measurement based on a photonic crystal fiber modalinterference interacting with a long period fiber grating," Opt. Commun., vol. 258, pp. 4874-4877, 2012.

[14] R. A. Perez-Herrera, R. M. André, S. F. Silva, M. Becker, K. Schsuster, J. Kobelke, M. Lopez-Amo, J. L. Santos, and O. Frazão, "Simultaneous measurement of strain temperature based on clover microstructured fiber loop mirror," Proc. SPIE, vol. 8421, p. 84216W-1 (4 pages), 2012.

[15] R. M. André, M. B. Marques, P. Roy, and O. Frazão, "Fiber loop mirror using a small core microstructured fiber for strain an temperature discrimination," IEEE Photon. Technol. Lett., vol. 22, no. 15, pp. 1120-1122, Aug. 2010.

[16] S. Liu, N. Liu, M. Hou, J. Guo, Z. Li, and P. Lu, "Direction-independent fiber inclinometer based on simplified hollow core photonic crystal fiber," Opt. Lett., vol. 38, pp. 449-451, 2013.

[17] B. Kim, T. H. Kim, L. Cui, and Y. Chung, "Twin core photonic crystal fiber for in-line Mach-Zehnder interferometric sensing applications," Opt. Exp., vol. 17, pp. 15502-15507, 2009.

[18] P. R. Horche, M. López Amo, M. A. Muriel, and J. A. Martín-Pereda, "Spectral behavior of a low cost all fiber component based on untapered multifiber unions," IEEE Photon. Technol. Lett., vol. 1, no. 7, pp. 184-187, Jul. 1989.

[19] S. Rota-Rodrigo, M. López-Amo, J. Kobelke, K. Schuster, J. L. Santos, and O. Frazão, "Simultaneous strain and temperature measure based on a single suspended core photonic crystal fiber," in Proc. 23rd Int. Conf. Opt. Fiber Sens., 2014, p. 915721 (4 pages).
[20] W. Jin, W. C. Michie, G. Thursby, M. Konstantaki, and B. Culshaw, "Simultaneous measurement of strain and temperature: Error analysis," Opt. Eng., vol. 36, pp. 598-609, 1997.

Sergio Rota-Rodrigo was born in Navarra, Spain, in October 1985. He received the Telecommunication Technical Engineering, Telecommunication Engineering and Communications Master degrees from the Universidad Pública de Navarra, Navarra, in 2007, 2010, and 2011, respectively. In 2011, he joined the Optical Communications Group, Department of Electrical and Electronic Engineering, Universidad Pública de Navarra. His research interests include fiber optic lasers, optical amplifiers, optical fiber sensor networks, photonic crystal fibers and chemical fiber optic sensors.

Manuel López-Amo (M'91-SM'98) was born in Madrid, Spain, in 1960. He received the Telecommunications Engineering and Ph.D. degrees from the Universidad Politécnica de Madrid, Madrid, in 1985 and 1989, respectively. From 1985 to 1996, he belonged to the Photonic Technology Department, Universidad Politécnica de Madrid, where, in 1990, he became an Associate Professor.

In 1996, he moved to the Electrical and Electronic Engineering Department, Public University of Navarra, Pamplona, Spain, where he became a Full Professor and is currently the Head of the Optical Communications Group. He has been the Chairman of the Optoelectronic Committee of Spain. He has been the leader of more than 40 research projects and he has coauthored more than 250 works in international refereed journals and conferences related with fiber-optic networks, optical amplifiers, fiber-optic sensors, and integrated optics. He is a member of the technical committees of the International Conference on Fiber Optic Sensors, the European Workshop on Optical Fiber Sensors, and European Cost TD1001 Action, among others. He is a Member of the Optical Society of America.

Jens Kobelke received the Ph.D. degree in chemistry from the Technische Hochschule Leuna, Merseburg, Germany, in 1986. Since this time, she works at IPHT Jena on the development and preparation of special optical fibers based on different glass materials (e.g., chalcogenide glasses, HMO glasses, high silica). Actual activities cover the fabrication technology and characterization of microstructured fibers based on high silica and other glass materials.

Kay Schuster has been engaged for many years in preparation of specialty fibers, based on heavy metal oxide glasses, chalcogenide glasses and high purity silica. His activities included the preparative operations of glass manufacturing as well as material characterization (DTA, TG, UV-VIS, FTIR spectroscopy, refractive index) and fiber fabrication (preform and crucible drawing). Based on his knowledge and experience both, special glass preparation and optical fiber drawing could be highly qualified at IPHT for different applications. Results of these activities were active nonsilica single mode fibers for amplification $\left(\mathrm{Pr}^{3+}\right.$ doped chalcogenide fibres for $1.3 \mu \mathrm{m}, \mathrm{Er}^{3+}$-doped HMO fibers for $1.5-\mu \mathrm{m}$ broadband amplification). His recent activities are concentrated on design and preparation of special functionalized microstructured fibers for passive, active and remote sensing applications. Very complex structures of this high silica and silicate glass-based fibers have been realized. In the applicative critical field of special sensor fibers for high temperature applications he is intensively engaged in the application and alignment of suitable fiber coating materials (e.g., ORMORCERs, siloxanes, polyimides). 
José Luis Santos received the Licenciatura in physics from the University of Porto, Porto, Portugal, and Ph.D. degree from the same university, benefiting from a collaboration with the University of Kent, Canterbury, U.K. He is currently a Professor of physics at the Physics and Astronomy Department, Faculty of Sciences, University of Porto. He is also Researcher of INESC Porto Optoelectronics and Electronic Systems Unit. Optical fiber sensing is the main area of his research, with focus on interferometric and wavelength-encoded devices. He is author or coauthor of more than 200 scientific articles and coauthor of five patents.
Orlando Frazão received the degree in physics engineering (optoelectronics and electronics) from the University of Aveiro, Aveiro, Portugal, and the Ph.D. degree in physics from the University of Porto, Porto, Portugal, in 2009. From 1997 to 1998, he was with the Institute of Telecommunications, Aveiro. He is currently a Senior Researcher with the Optoelectronics and Electronic Systems Unit, INESC Porto. He has published about 250 papers, mainly in international journals and conference proceedings, and his present research interests include optical fiber sensors and optical communications. He is a Senior Member of the Optical Society of America. 